\title{
Belphégor
}

Littérature populaire et culture médiatique

18-1 | 2020

Regards croisés sur la culture médiatique européenne

\section{Di Grado, Antonio. L'idea che uccide. I romanzieri dell'anarchia tra fascino e sgomento}

\section{Vittorio Frigerio}

\section{(2) OpenEdition}

1 Journals

\section{Electronic version}

URL: https://journals.openedition.org/belphegor/2113

DOI: 10.4000/belphegor.2113

ISSN: $1499-7185$

Publisher

LPCM

\section{Electronic reference}

Vittorio Frigerio, "Di Grado, Antonio. L'idea che uccide. I romanzieri dell'anarchia tra fascino e sgomento", Belphégor [Online], 18-1 | 2020, Online since 07 February 2020, connection on 29 June 2021. URL: http://journals.openedition.org/belphegor/2113 ; DOI: https://doi.org/10.4000/belphegor.2113

This text was automatically generated on 29 June 2021.

\section{(c) (i) (9)}

Belphégor est mis à disposition selon les termes de la Licence Creative Commons Attribution - Pas d'Utilisation Commerciale - Pas de Modification 4.0 International. 


\section{Di Grado, Antonio. L'idea che uccide. I romanzieri dell'anarchia tra fascino $e$ sgomento}

\section{Vittorio Frigerio}

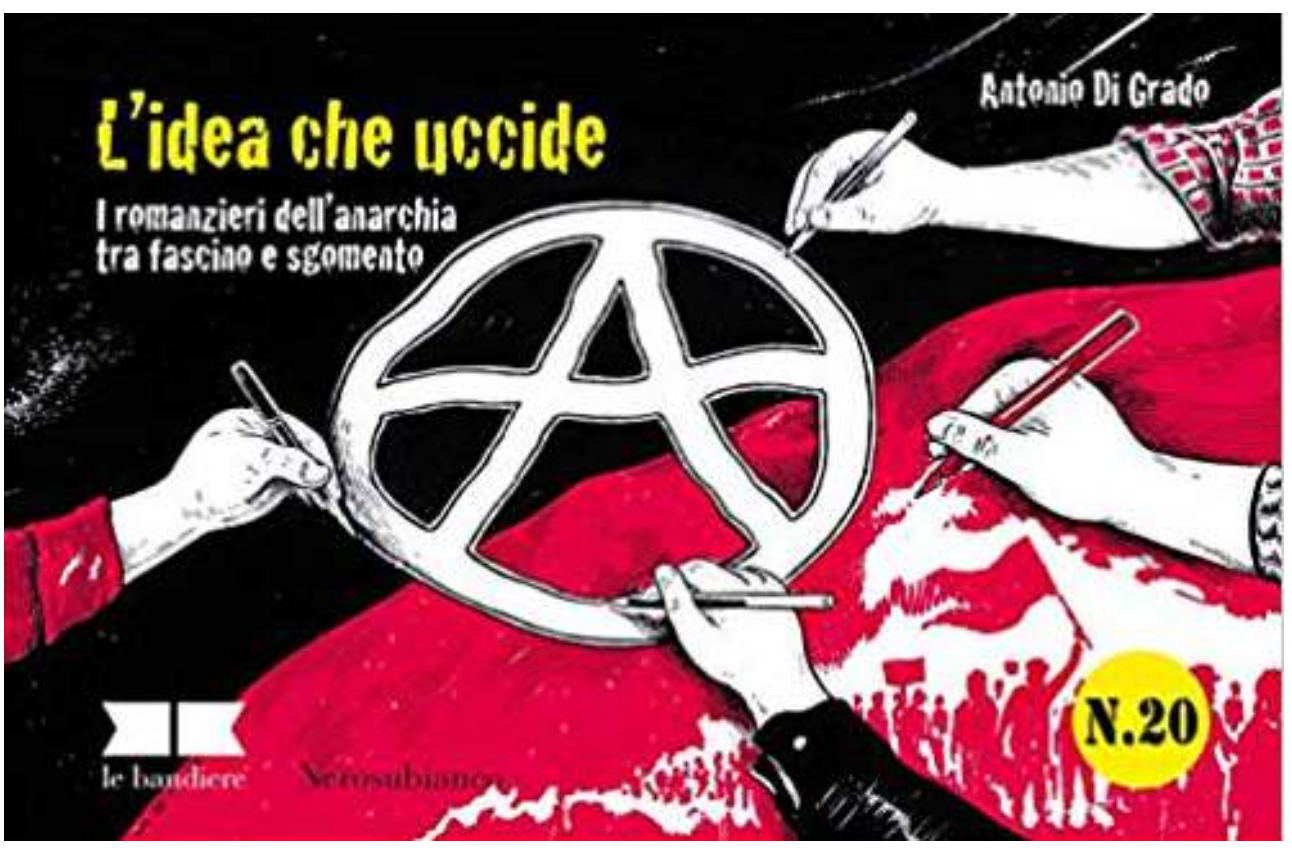

1 È verso la fine di questo agile e godibilissimo libretto che l'autore, Antonio Di Grado, sceglie di darci un breve riassunto della filosofia alla base del suo approccio alla storia lunga e complicata - dei rapporti tra letteratura e anarchia, esplicitando dei principî chiari che, arrivato a quel punto, anche il lettore distratto avrebbe potuto quanto meno intuire: "Questo mio scritto, se mi è consentita un'importuna intromissione, è una discutibilissima prova di quanto io esecri gli algidi teoremi della saggistica accademica d'impronta anglosassone e i post hoc, propter hoc d'uno storicismo corrivo, deterministico e unilineare. Ritengo assai più proficuo lasciar liberi i testi di fluttuare 
nell'universo illimitato delle analogie, di scegliersi remote e imprevedibili parentele anziché farsi ingabbiare nelle ferree griglie ermeneutiche d'un arrogante e prevenuto esegeta" (88). Fedele a questa visione - che ha il vantaggio di trasferire al metodo analitico le ispirazioni del tema trattato - Di Grado fornisce un tour d'horizon veloce e stimolante della presenza di idee o figure anarchiche nelle opere di autori principalmente, ma non esclusivamente, italiani, in generi che spaziano dal feuilleton dei bei tempi andati fino alla musica di Fabrizio De André, rivisitato in chiusura. Si ritrovano così al filo delle pagine nomi conosciuti e attesi, come quelli del De Roberto di Spasimo e dell'Imperio o del Bianciardi della Vita agra, ma anche d'illustri e affascinanti sconosciuti, quale "Clémentine Hugo, nipote aquisita del grande Victor" (28), o Antonio Agresti, autore d'un romanzo dimenticato (a quanto pare a torto) al quale il critico prende in prestito il titolo per il suo volume. Non poteva mancare Bacchelli, accompagnato da Zola, Bourget, Conrad e altri.

2 Ogni autore - è lo scotto da pagare in questo genere di saggio, vivace quanto veloce - è trattato rapidamente, talvolta nello spazio di qualche pagina, spesso anche in una sola. Ma la rapidità ha il vantaggio d'ispirare al critico formule giuste e sintetiche, che si tratti del semplice e azzecato aggettivo "sfortunatissimo" riservato ad Alfredo Oriani (42) - romanziere fin de siècle la cui fama ha ingiustamente sofferto dell'ammirazione postuma portatagli da Mussolini - o della magnifica definizione di Oblomov, il personaggio del romanzo eponimo di Ivan Gontcharov, bollato "santo taoista della nonazione" (51). Certuni degli autori trattati rientrano pienamente e a testa alta nel campo (poco arato) dell'anarchia letteraria, quale per esempio la sorprendente Leda Rafanelli, libertaria, femminista, chiromante e per finire convertita all'Islam, pur se in versione soft e sufi, sulla quale Nerosubianco aveva già attirato l'attenzione in due volumi della collana «le drizze» (Memorie d'una chiromante e I due doni e altre novelle orientali, entrambi a cura di Milva Maria Cappellini) e su cui è uscito da poco anche un fumetto, o piuttosto un "romanzo grafico" intitolato per l'appunto Leda Rafanelli: la gitane anarchiste, di Francesco Satta, Luca de Santis e Sara Colaone, che non rientra nell'indagine di Di Grado ma mostra la tenuta, nel nostro immaginario, di questa particolare artista innamorata della vita. Altri, come per esempio Georges Simenon, vi entrano invece di straforo, più che altro per una parentela ideale, fatta non di dichiarazioni o di idee ma di attitudini e di corrispondenze quasi baudleriane. E questo è il punto dove, volendo, si potrebbe trovare da ridire al lato volutamente 'ecumenico' della visione di Di Grado, che passa dalla chiara, "insostenibile coerenza dell'anarchico" (20) riconosciuto in quanto tale, autore o protagonista, a semplici "pulsioni esistenziali" (81) creatrici di parentele ideali ma spesso alquanto elastiche. Nelle schiere dei rivoltosi viene in tal modo arruolato anche il pessimista per eccellenza, Leopardi. E il critico, cosciente di quanto il ravvicinamento - che eppur sembra istintivamente corretto - sia problematico, aggira la questione ridefinendo il termine principale dell'analisi in funzione dell'esempio letterario che si è voluto affiancargli: "Vogliamo dunque parlare di 'anarchia' a proposito dell'ignaro Leopardi? Solo a costo di attribuire a quel temibile termine, piuttosto che il marchio di uno schieramento, la febbrile temperatura e l'impeto inerme di una dolente fraternità, di una debordante pietà, di un implacato antagonismo" (36). In modo simile lo ridefinirà ancora più tardi parlando di Simenon: "l'anarchia di cui in questo caso si tratta poco ha a che fare con qualsivoglia movimento recante questo nome; penso piuttosto a un impasto di mitezza e ironia, di estraneità e silenzio, di sconfinata libertà e felice incoerenza" (90). E in ciò, Di Grado si rivela perlomeno vicino a tanti critichi anarchici, autodidatti o poco meno, che sulle pagine di 
riviste come La Revue anarchiste o Les Temps Nouveaux pubblicavano liste allungabili a dismisura di illustri scrittori del passato promossi antenati nobili del movimento.

3 Ambiguità, frontiere porose, vicinanze scomode e di primo acchito 'insensate' eppure innegabili. Nell'ambito della letteratura italiana non si può, trattando di questo soggetto, ignorare l'ombra lunga del fascismo, movimento nel quale, alle origini perlomeno, confluirono figure vicine a posizioni anarchico-individualiste, come (in campo ben lontano dalla letteratura) quella di Leandro Arpinati, o altri rappresentanti di "una variante detta 'di sinistra', ribellistica e anti-borghese, su cui molto è ancora da dire per districare quel groviglio di generosi e velleitari azzardi” (76). Di Grado esplora a ragione le zone grige dove si aggirano autori quali Ungaretti, Pirandelllo, Malaparte, Savinio, perfino Vittorini, alle prese "con un fascismo non solo in attesa di verifica, ma irregolarmente saturo di elementi stirneriani e soreliani” (78). Si può parlare di "anarchismo di destra" (58), come é stato fatto per esempio per Léon Bloy? La questione resta aperta e in certi ambienti aspramente contesa, e il termine è sicuramente più che equivoco. Ma molto è appunto equivoco in queste no man's lands dell'ideologia, e piuttosto che di giungere a conclusioni categoriche, non si può spesso, come fa Di Grado nel caso di Vittorini, che ammettere di trovarsi di fronte ad una "irrisolta ambivalenza" (86) che va presa semplicemente come tale.

4 Ricco di riferimenti, di piccole scoperte, di parallelismi sorprendenti e talvolta discutibili ma sempre stimolanti, questo libro richiama alla mente una passeggiata - o un vagabondaggio - in gradevole e colta compagnia. È un po' così d'altronde che pare averlo concepito lo stesso Di Grado, evocando il gran flâneur parigino attraverso il suo esegeta più famoso e proponendo, con chiarezza e coerenza, un'esplorazione letteraria liberata dalle catene della cronologia. Lasciamo allora all'autore le mot de la fin, con il quale ci piace dirci idealmente in sintonia:

Ma forse tutta la storia delle idee e delle arti è tramata da rispondenze tanto più tenaci perché impossibili e animata da incontri mai avvenuti e tuttavia fatali, è illuminata da galassie che solo casualmente si condensano e poi si dissolvono nel momento in cui vorremmo fissarle, magari catalogarle e datarle (...).

Sono varchi che si aprono all'improvviso immettendo in un altrove che pareva irraggiungibile, cosí come i passages parigini che, secondo Benjamin, Fourier assunse a modello dei suoi falansteri. Quella storia, allora, non adrebbe più resa da puntuali resoconti di ambienti, scuole, relazioni, ma da fantasiose operette morali, da dialoghi lucianei nell'oltretomba. E converrebbe sbarazzarsi, finalmente, della greve coercizione delle concatenazioni causali, lasciando liberi autori e opere di volteggiare nel firmamento delle analogie, di scovarvi incontri e liaisons che non intrattennero in vita ma che a noi posteri possono dire altro o di più del pur vasto repertorio delle loro letture e frequentazioni. (34)

\section{AUTHOR \\ VITTORIO FRIGERIO}

Dalhousie University 